Proc. Indian Acad. Sci. (Earth Planet. Sci.). Vol. 92. Number 2. July 1983, pp. 99-104.

- Printed in India.

\title{
Temperature variation in the mesosphere during solar eclipse
}

\author{
P CHAKRABARTY, D K CHAKRABARTY and A K SAHA* \\ Physical Research Laboratory, Ahmedabad 380 009. India \\ * National Physical Laboratory. New Delhi 110 012, India \\ MS received 14 May 198I: revised 22 October 1982
}

\begin{abstract}
Rocket-borne observations of the extinction of solar hydrogen Lyman-alpha radiation made during the solar eclipses of 20 May 1966 at Karistos for normal and $44 \%$ visibility and of 7 March 1970 at East Quoddy for normal. 10\% and 0.6\% visibility have been used to study the variation of temperature in the mesosphere during these events. It is seen that near the mesopause the decrease of temperature at $\mathrm{Karistos}$ is by $20^{\circ} \mathrm{K}$ for $44 \%$ visibility and at East Quoddy by $100^{\circ} \mathrm{K}$ for $0.6 \%$ visibility. Possible causes of these temperature variations vis-a-vis molecular oxygen and pressure variations have been briefly discussed.
\end{abstract}

Keywords. Temperature; mesosphere: Lyman-alpha; solar eclipse.

\section{Introduction}

The temperature of the mesosphere, particularly of the mesopause, is a sensitive parameter which undergoes variations with any change in the geophysical condition of the ionosphere. While in phenomena like winter anomaly and polar cap absorption an increase of temperature has been reported (Thrane et al 1979; Faire and Murphy 1972), it is not known with certainty whether during solar eclipse the temperature undergoes any change in this region. The solitary measurement made so far by Horvath and Theon (1972) during the solar eclipse of 7 March 1970 by Pitot tube probes could not detect any change in temperature from that of a normal day. However, to explain the existence of the higher percentage of positive water cluster ions observed by Narcisi et al (1972) and Kopp (1979) during the solar eclipses of 12 November 1966 and 26 February 1979, a decrease in temperature of the mesopause region is a must. In the absence of any reliable information it would be worthwhile to examine the available extinction profiles of solar Lyman-alpha radiation measured during various solar eclipses and see what picture of variation of temperature of mesosphere emerges.

\section{Theory of temperature derivation}

If $n_{i}$ be the number density and $m_{i}$ the mass number of the $i$ th constituent in a layer of thickness $\mathrm{d} z$, then the change in the intensity $I$ of solar radiation as it traverses through the layer is given by

$$
\mathrm{d} I=I \sum n_{i} \sigma_{i} \sec \chi \mathrm{d} z
$$

where $\sigma_{i}$ is the absorption cross-section and $\chi$ is the solar zenith angle. Since $\mathrm{O}_{2}$ molecules are the dominant absorbers of Lyman-alpha, 


$$
\mathrm{d} I=I \cdot n\left(\mathrm{O}_{2}\right) \cdot(\sigma+f) \cdot \sec \chi \cdot \mathrm{d} z
$$

where $f$ is a small correction to account for other absorbers and its value is of the order of $5 \times 10^{-26} \mathrm{~m}^{2}$ (Hall 1972). If $\mathrm{d} p$ be the pressure difference accross the layer then

$$
\mathrm{d} p=-g \sum_{i} n_{i} m_{i} \mathrm{~d} z
$$

Combining (1) and (2) one obtains

$$
\mathrm{d} I / I=-(\sigma+f) \mathrm{d} p / K \cdot \cos \chi
$$

where $\quad K=g \sum_{i} n: m$ :inin. I

$K$ is constant for practical purposes at all heights. For monochromatic radiation

$$
I=I_{\infty} \exp \left(-\frac{(\sigma+f) \cdot p}{K \cdot \cos \chi}\right)
$$

From (1) one can write

$$
n\left(\mathrm{O}_{2}\right)=\frac{\cos \chi}{\sigma} \cdot \frac{1}{I} \cdot \frac{\mathrm{d} I}{\mathrm{~d} z}
$$

The parameters on the right side are all known and hence one can estimate $n\left(\mathrm{O}_{2}\right)$ from (3). From (2) one gets

$$
\mathrm{d} p / \mathrm{d} z=-K \cdot n\left(\mathrm{O}_{2}\right)
$$

Substituting for $n\left(\mathrm{O}_{2}\right)$ from (3) one gets

$$
\begin{gathered}
\mathrm{d} I / I=-\sigma \mathrm{d} p / K \cdot \cos \chi \\
\text { or } \quad I=I_{\infty} \exp \left(-\int \frac{\sigma \mathrm{d} p}{K \cos \chi}\right) .
\end{gathered}
$$

Hall (1972) has plotted $I / I_{\infty} v s p /\left(K, 10^{23} \cdot \cos \chi\right) \mathrm{m}^{-2}$. Once $p$ is known, one can calculate the scale height in terms of $p$ and $n\left(\mathrm{O}_{2}\right)$ which is given by

$$
\frac{1}{H}=-\frac{1}{p} \frac{\mathrm{d} p}{\mathrm{~d} z}
$$

Using (4) we get

$$
H=p / K \cdot n\left(\mathrm{O}_{2}\right)
$$

The scale height $H$ is also related to the temperature by the relation

$$
H=k T / m g
$$

and hence $T$ can be estimated. 


\section{Data}

Rocket observations of the extinction of solar hydrogen Lyman-alpha radiation have been used by several workers to derive the temperature of the mesosphere (Thrane et al 1979; Thrane and Johannessen 1975; Hall 1972) under various geophysical conditions. Hall (1972) studied this method in detail and demonstrated that, if the absorption cross-section of Lyman-alpha in molecular oxygen is known, then this technique can give the values of pressure. density and temperature of the mesosphere with good accuracy. While many workers have used a constant value of absorption cross-section. Hall (1972) points out that it is essential to take into account the variation of this parameter with wavelength across the $0.1 \mathrm{~nm}$ wide solar line. Thrane and Johannessen (1975) find that the laboratory measurements of the absorption cross-sections of Lyman-alpha in molecular oxygen do not apply to conditions in the upper atmosphere. The scope of this work is not to examine the correctness of this absorption cross-section value but to use the same value of absorption cross-section to the Lyman-alpha extinction profiles measured during and out of eclipse conditions (Hall 1970; Dickinson 1972) in two solar eclipse events and estimate the temperatures for both the conditions. One of the events occurred on 20 May 1966 over Karistos, Greece $\left(38^{\circ} \mathrm{N}, 24^{\circ} \mathrm{W}\right)$ where Lyman-alpha extinctions were measured during normal and $44 \%$ visibility conditions. The other eclipse event took place in East Quoddy. Canada $\left(45^{\circ} \mathrm{N}, 62^{\circ} \mathrm{W}\right)$ where four rockets were launched during normal, $0.15 \%, 0.6 \%$ and $10 \%$ visibility condition. The last two rockets measured after the eclipse totality i.e. in the regaining phase of the ionisation. There was considerable error in the $0.15 \%$ eclipse totality measurements (Dickinson 1972) and hence we have not used this measurement in our present work. Using these profiles, the temperature values of the mesosphere have been derived by the method described by Hall (1972) and which has been described briefly above. It takes into account the variation of absorption crosssection across the Lyman-alpha line. Calculations have been done at an interval of $1 \mathrm{~km}$.

\section{Results}

In figure 1 , the temperature values during normal and different visibility conditions have been plotted for both the solar eclipse events. It shows that at Karistos the decrease is maximum by about $20^{\circ} \mathrm{K}$ in the region $80-83 \mathrm{~km}$. At East Quoddy the maximum decrease by $100^{\circ} \mathrm{K}$ is around $80-83 \mathrm{~km}$ when the visibility decreased from full sun to $0.6 \%$. An interesting point is the near similarity of the shapes of temperature profiles of flight 4 which is in the regaining phase of ionization and flight 1 which is in the full sun condition. Also. of the three temperature profiles obtained for the East Quoddy eclipse, the temperature profile of flight 3 shows a minimum around $80-82 \mathrm{~km}$ while the other two temperature profiles for flights 1 and 4 show a bulge around this height. A temperature bulge around the mesopause level for normal conditions was reported earlier (Chakrabarty and Chakrabarty 1979) for Wallops Island where the temperature was estimated from rocket measurements of $\left[\mathrm{NO}^{+}\right]$/ $\left[\mathrm{NO}^{+} \mathrm{H}_{2} \mathrm{O}\right]$ ratio.

The accuracy of the temperature estimations depend critically on the reliability with which the Lyman-alpha extinction profiles a re measured during these events. The sensitivity of the instruments is a parameter which may not remain constant during and out of eclipse conditions. The principal source of error in the flux determination is contamination of the chamber window. Dickinson (1972), however, puts the overall 


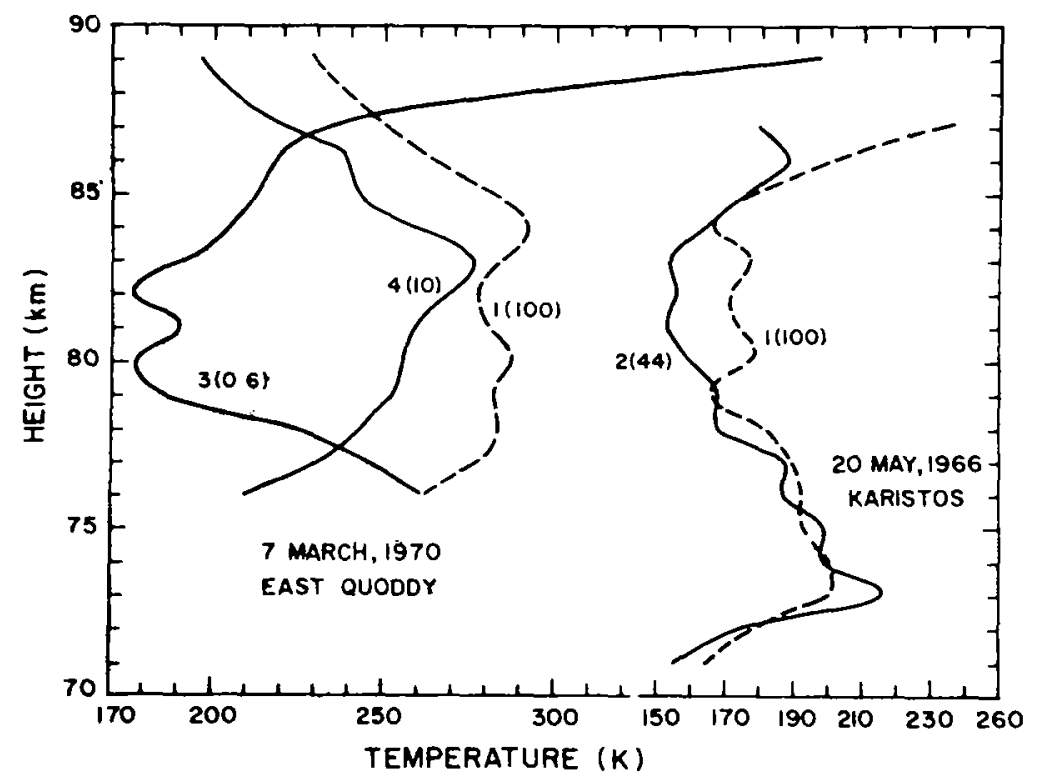

Figure 1. Temperature profiles estimated from Lyman-alpha extinction measurements during the solar eclipses of 20 May 1966 and 7 March 1970 at Kaistos. Greece and East Quoddy. Canada. Numbers inside the parentheses denote visibility in $\%$ and the numbers outside denote the sequence of rocket flights.

uncertainty in the absolute calibration of East Quoddy measurements to about $\pm 20 \%$. In the present work, since we have considered the ratio $\mathrm{d} I / I$, the overall error in estimated temperature would be less than $\pm 20 \%$. It may be mentioned here that the two measurements of Lyman-alpha flux made by oso-5 satellite at an interval of about $3 \mathrm{hr}$ are available for $7 \mathrm{March} 1970$ (Vidal-Madjer 1975). These values are 5.7 ergs $\mathrm{cm}^{-2} \mathrm{sec}^{-1}$ in agreement with the normal time value of $5.3 \mathrm{ergs} \mathrm{cm}^{-2} \mathrm{sec}^{-1}$ measured by Dickinson (1972) at East Quoddy.

\section{Discussion}

It is known that in the $D$-region during solar eclipse the degree of hydration of proton hydrates increases (Kopp 1979; Narcisi et al 1972). An explanation of this feature demands a decrease in temperature during solar eclipse. The temperature estimates in figure 1 have been computed from the scale height of molecular oxygen. The scale height is related to the density of molecular oxygen and pressure by equation (7). Hence any variation of scale height could be due to changes in the pressure and molecular oxygen density. The molecular oxygen density has been estimaed by Hall (1970) (with constant $\sigma$ ) for the 20 May 1966 eclipse at Ka ristos. He concluded that the oxygen densities were slightly lower on the eclipse day but the difference is barely significant. We have repeated these calculations of molecular oxygen with $\sigma$ values from Hall (1972) (in which $\sigma$ as a function of wavelength has been assumed) for the eclipse events and no significant changes are seen in the molecular oxygen density for the eclipse event at Karistos. For the eclipse event of East Quoddy, however, we see an increase of molecular oxygen density. These are shown in figure 2 . The other parameter on which the scale height is dependent is the pressure which also changes during eclipse. The question which arises, therefore, is how does eclipse phenomenon cause a 


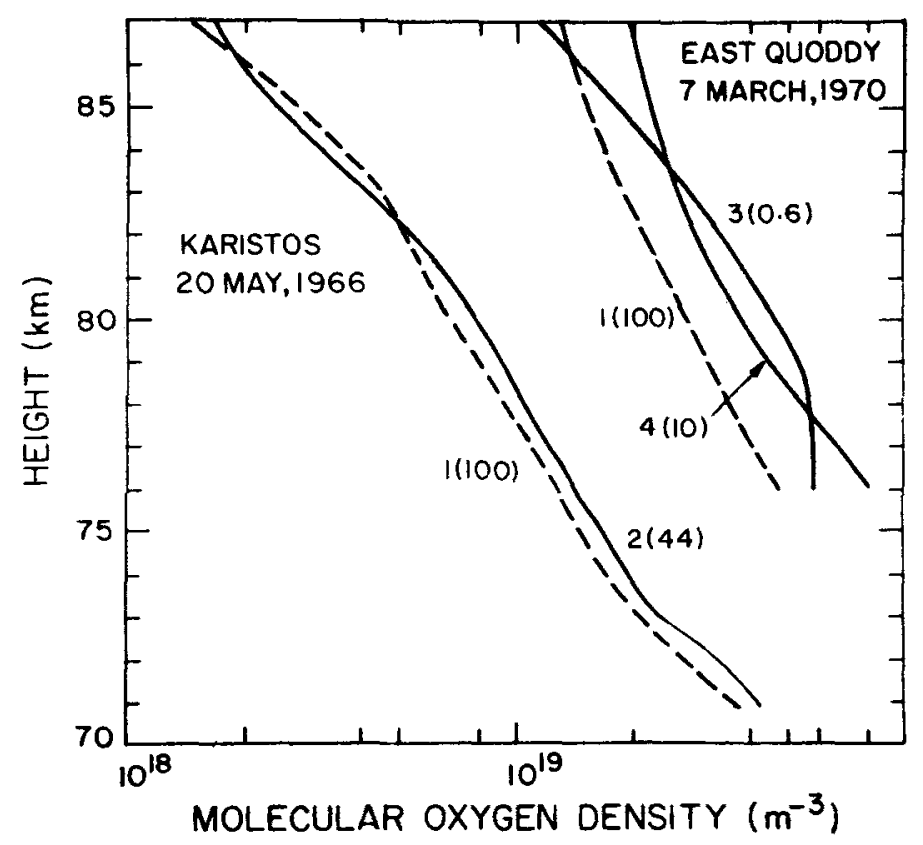

Figure 2. Molecular oxygen density profiles estimated from Lyman-alpha extinction measurements during the solar eclipses of 20 May 1966 and 7 March 1970 at Karistos, Greece and East Quoddy. Canada.

variation in pressure and molecular oxygen density? The two plausible reasons are dynamical and solar radiation effects. It is likely that the supersonic motion of the moon's shadow triggers wave motion causing compression and rarefaction in the whole length of the atmosphere. Gravity waves generated by the shadow of moon as it moves supersonically across the earth's atmosphere had been predicted long ago by Chimonas and Hines (1970). Total electron content observations at four stations in western United States during the solar eclipse of 7 March 1970 detected travelling disturbances which possibly confirmed the suggestion of Chimonas and Hines 1971; Davis and da Rosa 1970. However, TIDs observed in a number of ionospheric experiments in United States during the same period were not conclusively attributed to the eclipse effect (Carlson et al 1970; Larford et al 1972; Arendt 1972; Sears 1972). From observations of Faraday rotation and group delay measurements at $40 \mathrm{MHz}$ using radio beacons from ATS-6 at Trivandrum, Vaidyanathan et al (1978) had reported quasi-periodic fluctuations of about 10 min periodicities during the partial eclipse of 29 April 1976. However, Sethia et al (1980) using the ATS-6 satellite data for a number of other locations for the same eclipse reported absence of travelling ionospheric disturbances at all these stations. Thus we see that the presence/absence of eclipse-induced gravity waves is still highly controversial

An alternate reason of the change in the pressure and molecular oxygen.density could be the absorption or generation of heat at different regions of the atmosphere when Sun's disc starts getting obscured by the moon. A theoretical calculation has shown that while at stratopause a cooling is produced (by about $10^{\circ} \mathrm{K}$ ), at the tropopause region an increase of temperature is noticed (Davies 1981) during a solar eclipse. A detailed theoretical computation of temperature could show whether the mesopause will also be cooled along with stratopause during solar eclipse. 


\section{Conclusion}

Mesopause temperatures are derived from the rocket-borne observations of Lymanalpha extinction profiles made during the solar eclipses of 20 May 1966 at Karistos and of 7 March 1970 at East Quoddy. At both places a decreasing trend is noticed. The accuracy of these estimations depends critically on the reliability with which the Lyman-alpha extinction profiles are measured during these events. The temperature va riations may be due to either some sort of waves generated by the supersonic motion of the shadow of the moon across the earth's atmosphere or absorption generation of heat at various layers of the earth's atmosphere as the solar disc is rapidly covered by the moon.

\section{Acknowledgements}

Our grateful thanks to Drs A P Mitra, K Davies and C A Reddy for fruitful discussions during the International Solar Eclipse Symposium in New Delhi, India in January 1981. Thanks are also due to Dr H Chandra for discussions on eclipseinduced gravity waves. One of the authors (PC) acknowledges with thanks the Director, Physical Research Laboratory, Ahmedabad for financial assistance.

\section{References}

Arendt P R 1972 J. Atmos. Terr. Physs. 34719

Carlson H C. Herper R. Wickard V. Showen R L. Behnke R. Trost T F. Cogger L R and Nelson C R 1970 Narure (London) 2261124

Chakrabarty P and Chakrabarty D K $1979 \mathrm{~J}$. Geophys. Res. 843403

Chimonas G and Hines C O $1970 \mathrm{~J}$. Geophys. Res. 75875

Chimonas G and Hines C O 1971 J. Geophys. Res. 767003

Davies K 1981 Paper presented at the Int. Solar Eclipse Symp., New Delhi. January

Davis M J and da Rosa A V 1970 Nature (London) 2261123

Dickinson P H G 1972 J. Atmos. Terr. Phys. 34621

Faire A C and Murphy E A 1972 Solar particle events of November 1969. Proc. COSPAR Symp.. AFCRL-72-0474. Special Report No. 144. p. 445

Hall J E $1970 \mathrm{~J}$. Atmos. Terr. Phis. 321849

Hall J E $1972 J$. Almos. Terr. Phy's. 341337

Horvath J J and Theon J S 1972 J. Atmos. Terr. Phy's. 34593

Kopp E 1979 Private Communication

Larford G M, Jurgens R M. Vesscky J F and Warhbann T W 1972 J. Atmos. Terr. Phis. 34733

Narcisi R S. Bailey A D. Wlodyka L. E and Philbrick C R 1972 J. Atmos. Terr. Phys. 34647

Sears R D $1972 J$. Atmos. Terr. Phys. 34727

Sethia G. Chandra H and Deshpande M R 1980 Proc. Indian Acad. Sci.(Earth Planet. Sci.) 89153

Thrane E V and Johannessen A 1975 J. Almos. Terr. Phys. 37655

Thrane E V. Grandal B. HogenO, Uglet Veit F. Bangert W. Beran D. Friedrich M, Loidl A, Schwentek H and Torkar K M 1979 J. Almos. Terr. Phys. 411097

Vaidyanathan S. Raghava Reddi C and Krishna Murthy B V 1978 Nature (London) 27141

Vidal-Madjer A 1975 Solar Phy's. 4069 